\title{
Categorical crepant resolutions for quotient singularities
}

\author{
Roland Abuaf ${ }^{1}$
}

Received: 20 June 2014 / Accepted: 27 April 2015 / Published online: 23 November 2015

(C) The Author(s) 2015. This article is published with open access at Springerlink.com

\begin{abstract}
We discuss some results on categorical crepant resolutions for varieties with quotient singularities. Namely, we prove that under appropriate hypotheses, the derived category of a smooth Deligne-Mumford stack is a strongly crepant non-commutative resolution of singularities of its coarse moduli space.
\end{abstract}

\section{Contents}

1 Introduction . . . . . . . . . . . . . . . . . . . . . . . . . . . . 679

2 Categorical crepant resolutions of singularities . . . . . . . . . . . . . . . . . . . . . 681

3 Main results and proofs . . . . . . . . . . . . . . . . . . . . . . . . . . 683

4 Some connections with the McKay correspondence . . . . . . . . . . . . . . . . . . . . 687

References . . . . . . . . . . . . . . . . . . . . . . . . 688

\section{Introduction}

Let $X$ be an algebraic variety over $\mathbb{C}$. Hironaka proved that one can find a proper birational morphism $\tilde{X} \rightarrow X$, with $\tilde{X}$ smooth. Such an $\tilde{X}$ is called a resolution of singularities of $X$. Unfortunately, given an algebraic variety $X$, there is, in general, no minimal resolution of singularities of $X$. In case $X$ is Gorenstein, a crepant resolution of $X$ (that is a resolution $\pi: \tilde{X} \rightarrow X$ such that $\pi^{*} \omega_{X}=\omega_{\tilde{X}}$ ) is often considered to be minimal. Indeed, if $\tilde{X}^{\prime} \rightarrow X$ is another resolution of $X$ with a commutative diagram:

Supported by EPSRC programme Grant EP/G06170X/1.

$\triangle$ Roland Abuaf

r.abuaf@imperial.ac.uk

1 Imperial College London, South Kensington Campus, London SW7 2AZ, UK 


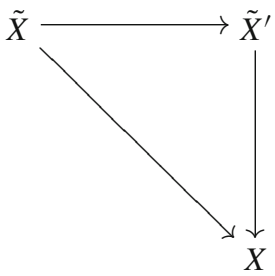

the ramification formula for proper birational morphisms between smooth varieties implies that $\tilde{X}=\tilde{X}^{\prime}$. Bondal and Orlov conjectured that a much stronger minimality property for crepant resolutions would hold at the categorical level (see [5]):

Conjecture 1.0.1 Let $X$ be an algebraic variety with canonical Gorenstein singularities. Assume that $X$ has a crepant resolution of singularities $\tilde{X} \rightarrow X$. Then, for any other resolution of singularities $Y \rightarrow X$, there exists a fully faithful embedding:

$$
\mathrm{D}^{\mathrm{b}}(\tilde{X}) \hookrightarrow \mathrm{D}^{\mathrm{b}}(Y)
$$

Varieties admitting a crepant resolution of singularities are quite rare. For instance, nonsmooth Gorenstein $\mathbb{Q}$-factorial terminal singularities (e.g. $\mathbb{C}^{2 n} / \mathbb{Z}_{2}$, for $n \geq 2$ ) never admit crepant resolution of singularities. Thus, it seems natural to look for minimal resolutions among categorical ones. Kuznetsov has given a definition of categorical crepant resolution of singularities which seems very well-fit to deal with the issue of minimal categorical resolutions of singularities (see [14]).

The goal of this short note is to prove some "folklore" results which concern categorical crepant resolutions of singularities for varieties with quotient singularities. The first one asserts that a global quotient always admits a categorical strongly crepant resolution which is non-commutative in the sense of Van den Bergh (see [20] for a definition of non-commutative resolution):

Theorem 1.0.2 Let $V$ be a smooth quasi-projective variety and let $\mathrm{G}$ be a finite subgroup of $\operatorname{Aut}(V)$. Assume that the dualizing sheaf of $V$ is $\mathrm{G}$-equivariantly locally trivial. Then $\mathrm{D}_{\mathrm{G}}(V)$, the derived category of G-equivariant quasi-coherent sheaves on $V$, is a categorical strongly crepant resolution of $V / \mathrm{G}$.

Furthermore, there exists a sheaf of algebras $\mathscr{A}$ on $V / \mathrm{G}$ such that $\mathrm{D}^{\mathrm{b}}{ }_{\mathrm{G}}(V) \simeq$ $\mathrm{D}^{\mathrm{b}}(V / \mathrm{G}, \mathscr{A})$. Hence, the pair $(V / \mathrm{G}, \mathscr{A})$ is a non-commutative crepant resolution of $V / \mathrm{G}$ in the sense of Van den Bergh.

The definition of strongly crepant categorical resolution will be given in Sect. 2 of this paper. It is a categorical analogue of the geometric notion of crepant resolution of singularities.

Corollary 1.0.3 Let $V$ be a finite dimensional vector space and let $\mathrm{G}$ be a finite subgroup of $\mathrm{SL}(V)$. Then $X / \mathrm{G}$ admits a non-commutative crepant resolution in the sense of Van den Bergh.

Corollary 1.0.3 is well-known and appeared already many times in the literature (see [20]). Some cases of Theorem 1.0.2 were already worked out by Kuznetsov [14]. Our second result deals with the global case:

Theorem 1.0.4 Let $X$ be a quasi-projective variety with normal Gorenstein quotient singularities and let $\mathscr{X}$ be a smooth Deligne-Mumford stack whose coarse moduli space is $X$. 
Assume that the dualizing line bundle of $\mathscr{X}$ is the pull-back of the dualizing bundle of $X$, then $\mathrm{D}(\mathscr{X})$ is a categorical strongly crepant resolution of $X$.

Furthermore, there exists a sheaf of algebras $\mathscr{A}$ on $X$ such that $\mathrm{D}^{\mathrm{b}}(\mathscr{X}) \simeq \mathrm{D}^{\mathrm{b}}(X, \mathscr{A})$. Hence, the pair $(X, \mathscr{A})$ is a non-commutative crepant resolution of $X$ in the sense of Van den Bergh.

Theorem 1.0.2 is a consequence of Theorem 1.0.4. However, I can give a proof of the former which is very low-tech and very direct, whereas the proof of the latter involves some delicate results about Deligne-Mumford stacks. Thus, I believe that it is interesting for the non-expert reader to have both results stated and proved separately.

In the last part of this paper, I will compare the strongly crepant resolution discussed above and the derived category of the classical resolution of a variety with scalar cyclic singularities.

Theorem 1.0.5 Let $\mathrm{G}$ be a cyclic group of order $d$ acting on $\mathbb{C}^{n}$ by translations (with $d$ dividing $n$ ). Denote by $\widehat{\mathbb{C}^{n} / \mathrm{G}}$ the resolution of singularities of $\mathbb{C}^{n} / \mathrm{G}$ obtained by blowing up 0. There is fully faithful embedding:

$$
\mathrm{D}_{\mathrm{G}}^{\mathrm{b}}\left(\mathbb{C}^{n}\right) \hookrightarrow \mathrm{D}^{\mathrm{b}}\left(\widetilde{\mathbb{C}^{n} / \mathrm{G}}\right) .
$$

In particular, if $d=n$, then $\widetilde{\mathbb{C}^{n} / \mathrm{G}}$ is a crepant resolution of $\mathbb{C}^{n} / \mathrm{G}$ and there is an equivalence:

$$
\mathrm{D}_{\mathrm{G}}^{\mathrm{b}}\left(\mathbb{C}^{n}\right) \simeq \mathrm{D}^{\mathrm{b}}\left(\widetilde{\mathbb{C}^{n} / \mathrm{G}}\right)
$$

This last result can be seen as an instance of the McKay correspondence for scalar cyclic singularities. As already noticed by Bridgeland, King and Reid, such an equivalence can not be proved using the main result of [4], when $n \geq 4$.

The three aforementioned results were expected to hold true by some experts and I claim no originality for them. For instance, Bridgeland [6] proves a special case of Theorem 1.0.5 and Kawamata ([11]) asks if there exists "interesting" (probably meaning crepant) categorical resolutions for varieties with quotient singularities. In [11], example 3.2, he proves a special case of the first part of Theorem 1.0.4 for $G=\mathbb{Z}_{2}$. He also expected that the first part of Theorem 1.0.4 should be true in general.

\section{Categorical crepant resolutions of singularities}

In this section, I remind some basic facts about categorical crepant resolution of singularities and we exhibit some classical examples as they appear in [14]. If $X$ is an algebraic variety over $\mathbb{C}$, we denote by $\mathrm{D}(X)$ the (unbounded) derived category of quasi-coherent sheaves on $X$. We let $\mathrm{D}^{\mathrm{b}}(X)=\mathrm{D}^{\mathrm{b}}(\operatorname{Coh}(X))$ be the bounded derived category of coherent sheaves on $X$.

Definition 2.0.6 ([12]) Let $X$ be an algebraic variety. A categorical resolution of $X$ is a smooth cocomplete compactly generated category $\mathscr{T}$ with a pair of adjoint functors:

$$
\begin{aligned}
& \pi_{*}: \mathscr{T} \rightarrow \mathrm{D}(X) \\
& \pi^{*}: \mathrm{D}(X) \rightarrow \mathscr{T},
\end{aligned}
$$

such that

- $\pi_{*} \pi^{*} \simeq i d$, 
- $\pi^{*}$ and $\pi_{*}$ commute with arbitrary direct sums,

- $\pi_{*}\left(\mathscr{T}^{c}\right) \subset \mathrm{D}^{\mathrm{b}}(X)$, where $\mathscr{T}^{c}$ is the subcategory of compact objects in $\mathscr{T}$.

I will not discuss the details of this definition and rather refer to [12] where the theory is developed with great care. In [14], the notion of categorical resolution was defined for the bounded derived category of coherent sheaves on $X$. In that case, the left adjoint to $\pi_{*}$ is only well defined on $\mathrm{D}^{\text {perf }}(X)$, which happens to be not convenient. This is one reason which explains the use of unbounded derived categories in the above definition. The following lemma makes the link between the definition in [14] and the one above:

Lemma 2.0.7 Let $X$ be a quasi-projective variety with rational singularities and let $q: \tilde{X} \rightarrow$ $X$ be a resolution of singularities. Let $\mathscr{T}_{0}$ be an admissible full subcategory of $\mathrm{D}^{\mathrm{b}}(\tilde{X})$ such that $\mathbf{L} q^{*} \mathrm{D}^{\text {perf }}(X)$ embeds fully and faithfully inside $\mathscr{T}_{0}$. If $\mathscr{T}$ is the completion of $\mathscr{T}_{0}$ inside $\mathrm{D}(\tilde{X}), \delta: \mathscr{T} \hookrightarrow \mathrm{D}(\tilde{X})$ the fully faithful embedding and $\delta^{*}$ the left adjoint to $\delta$, then

$$
\begin{aligned}
& \boldsymbol{R} q_{*} \circ \delta: \mathscr{T} \rightarrow \mathrm{D}(X) \\
& \delta^{*} \circ \boldsymbol{L} q^{*}: \mathrm{D}(X) \rightarrow \mathscr{T},
\end{aligned}
$$

is a categorical resolution.

Proof Since $\tilde{X}$ is a smooth quasi-projective variety, $\mathrm{D}(\tilde{X})$ is cocomplete, compactly generated ([15]) and smooth ([19], lemma 3.27). But $\mathscr{T}$ is the completion of an orthogonal component of $\mathrm{D}^{\mathrm{b}}(\tilde{X})$, so that $\mathscr{T}$ is also cocomplete, compactly generated and smooth ([12], for instance).

Let me denote by $\pi_{*}$ the composition $\mathbf{R} q_{*} \circ \delta$. We have $\pi_{*} \pi^{*} \simeq i d$ because $\mathbf{L} q^{*} D^{\text {perf }}(X)$ embeds fully and faithfully in $\mathscr{T}_{0}$ and $X$ has rational singularities. The functor $\pi^{*}$ always commutes with arbitrary direct sums whereas the functor $\pi_{*}$ commutes with arbitrary direct sums because it is right adjoint to $\pi^{*}$ and $\mathscr{T}$ is compactly generated. Finally, the inclusion $\pi_{*}\left(\mathscr{T}^{c}\right) \subset \mathrm{D}^{\mathrm{b}}(X)$ is a consequence of the properness of $q$.

Note that if $X$ has irrational singularities and $q: \tilde{X} \rightarrow X$ is a resolution of singularities, then $\mathbf{R} q_{*}: \mathrm{D}(\tilde{X}) \rightarrow \mathrm{D}(X)$ is NOT a categorical resolution of singularities. Nevertheless, we still have the following:

Theorem 2.0.8 ([12]) Any quasi-projective scheme (!) of finite type over $\mathbb{C}$ admits a categorical resolution of singularities.

I refer to [12] for interesting comments about this nice result and for a proof of it. In this note, we will focus on a very special type of categorical resolutions, the so-called categorical crepant resolutions of singularities. These are categorical resolutions which mimick very well the functorial behavior one expects from the derived category of a (geometric) crepant resolution of singularities. The main point of this theory is that we can sometimes construct categorical crepant resolutions even if the singularity has no geometric crepant resolution. This highlights a new point of view on the Minimal Model Program ([11,14]).

Definition 2.0.9 Let $X$ be an algebraic variety and let $\pi_{*}: \mathscr{T} \rightarrow \mathrm{D}(X)$ be a categorical resolution of $X$. The category $\mathscr{T}$ is said to be a weakly crepant resolution of $Y$ if $\pi^{*}$ is also a right adjoint to $\pi_{*}$ when restricted to $\mathrm{D}^{\text {perf }}(X)$.

Assume finally that $\mathscr{T}$ has a module structure over $X$. Then $\mathscr{T}$ is said to be a strongly crepant resolution of $X$ if the identity is a relative Serre functor for $\mathscr{T}^{c}$ with respect to $\mathrm{D}^{\mathrm{b}}(X)$. 
The notions of module structure and of relative Serre functor are defined in [14] for instance and they are as natural as one can imagine. Note however that if the categorical resolution is of "geometric origin", then all these notions coincide with the classical definition of crepancy. Namely, we have the proposition ([1], prop. 1.2.12):

Proposition 2.0.10 Let $\pi: \tilde{X} \rightarrow X$ be a morphism of algebraic varieties. Then:

$$
\begin{aligned}
& \boldsymbol{R} \pi_{*}: \mathrm{D}(\tilde{X}) \rightarrow \mathrm{D}^{\mathrm{b}}(X) \text { is a categorical strongly crepant resolution of singularities } \\
& \boldsymbol{R} \pi_{*}: \mathrm{D}(\tilde{X}) \rightarrow \mathrm{D}^{\mathrm{b}}(X) \text { is a categorical weakly crepant resolution of singularities } \\
& \qquad: \tilde{X} \rightarrow X \text { is a crepant resolution of singularities. }
\end{aligned}
$$

The existence of weakly crepant resolutions of singularities has been proved in a quite general context (see [2,3]). For instance, it is proved in [2] that all Gorenstein determinantal varieties (general, symmetric, skew-symmetric) admit weakly crepant resolution of singularities. The existence of strongly crepant resolution seems to be a much more delicate issue.

In [14], Kuznetsov proved the existence of categorical strongly crepant resolutions of singularities for scalar cyclic quotient singularities. Let me summarize how he found them, as I show in the last section of this paper that they are equivalent to the general construction I provide for quotient singularities. Recall that a (local) scalar cyclic quotient singularity is the data of a cyclic group $\mathrm{G}$ which acts by dilations on $\mathbb{C}^{n}$. It is well-known that the quotient $\mathbb{C}^{n} / \mathrm{G}$ is Gorenstein if and only if the order of $\mathrm{G}$ (say $d$ ) divides $n$ and that $\mathbb{C}^{n} / \mathrm{G}$ admits a crepant resolution of singularities if and only if $d=n$ (see [8]). In the following, I always assume that $d$ divides $n$.

Let me denote $X:=\mathbb{C}^{n} / \mathrm{G}$ and $q: \tilde{X} \rightarrow X$ the blow-up of $X$ at 0 . The morphism $q$ is a resolution of singularities and the exceptional divisor $i: E \hookrightarrow \tilde{X}$ is isomorphic to $\mathbb{P}^{n-1}$ with $\mathscr{O}_{E}(E)=\mathscr{O}_{\mathbb{P}^{n-1}}(-d)$. Note that $\tilde{X}$ is the total space of $t: \mathscr{O}_{\mathbb{P}^{n-1}}(-d) \rightarrow \mathbb{P}^{n-1}$.

Theorem 2.0.11 ([14]) There is a semi-orthogonal decomposition:

$$
\mathrm{D}^{\mathrm{b}}(\tilde{X})=\left\langle i_{*}\left(\mathscr{B} \otimes \mathscr{O}_{E}\left(\left(\frac{n}{d}-1\right) E\right)\right), \ldots, i_{*}\left(\mathscr{B} \otimes \mathscr{O}_{E}(E)\right), \mathscr{T}_{0}\right\rangle,
$$

where $\mathscr{B}=\left\langle\mathscr{O}_{\mathbb{P}^{n-1}}((1-d)), \ldots, \mathscr{O}_{\mathbb{P}^{n-1}}(-1), \mathscr{O}_{\mathbb{P}^{n-1}}\right\rangle$.

The completion of $\mathscr{T}_{0}$ in $\mathrm{D}(\tilde{X})$ is a categorical strongly crepant resolution of $X$ and there is an equivalence:

$$
\mathscr{T}_{0} \simeq \mathrm{D}^{\mathrm{b}}\left(X, q_{*} \mathscr{E} n d(\mathscr{A})\right),
$$

where $\mathscr{A}=t^{*} \mathscr{O}_{\mathbb{P}^{n-1}} \oplus t^{*} \mathscr{O}_{\mathbb{P}^{n-1}}(-1) \oplus \cdots \oplus t^{*} \mathscr{O}_{\mathbb{P}^{n-1}}(1-d)$.

\section{Main results and proofs}

In this section, I will prove Theorems 1.0.2 and 1.0.4 and some related corollaries.

Theorem 3.0.12 Let $V$ be a smooth quasi-projective variety and let $\mathrm{G}$ be a finite subgroup of $\operatorname{Aut}(V)$. Assume that the dualizing sheaf of $V\left(\right.$ denoted $\left.\omega_{V}\right)$ is G-equivariantly locally trivial, then $\mathrm{D}_{\mathrm{G}}(V)$ is a categorical strongly crepant resolution of $V / \mathrm{G}$. 
Furthermore, there exists a sheaf of algebras $\mathscr{A}$ on $X / \mathrm{G}$ such that $\mathrm{D}^{\mathrm{b}}{ }_{\mathrm{G}}(V) \simeq$ $\mathrm{D}^{\mathrm{b}}(V / \mathrm{G}, \mathscr{A})$. Hence, the pair $(X / \mathrm{G}, \mathscr{A})$ is a non-commutative crepant resolution of $X / \mathrm{G}$ in the sense of Van den Bergh.

The dualizing line bundle of $V$ being G-equivariantly trivial means that for each $v \in V$, one can find a G-stable open neighborhood of $v \in V$ such that $\omega_{V}$ restricted to that open is G-equivariantly trivial. This implies in particular that if $v \in V$ is a fixed point of the action on $\mathrm{G}$ on $V$, then the representation of $\mathrm{G}_{v}$ on $\omega_{V, v}$ is trivial. Hence, by descent theory for quotient maps by finite groups, we find that $V / \mathrm{G}$ is Gorenstein and that $\omega_{V}$ is the pull-back of $\omega_{V / \mathrm{G}}($ see [17]).

Proof The category $\mathrm{D}_{\mathrm{G}}(V)$ is obviously cocomplete. Since $V$ is quasi-projective, it admits a G-equivariant ample line bundle, say $L$. The same assertions as in [15], example 1.10, carry over to show that if $V_{\rho_{1}}, \ldots, V_{\rho_{p}}$ are the finite irreducible representations of $\mathrm{G}$, then the family:

$$
\left\{L^{\otimes m}[n] \otimes V_{\rho_{l}}\right\}_{n, m \in \mathbb{Z}}^{l=1 \ldots p}
$$

generates $\mathrm{D}_{\mathrm{G}}(V)$. Finally, since $V$ is smooth and $\mathrm{D}_{\mathrm{G}}(V)$ is compactly generated, the same argument as in ([19], Lemma 3.27) show that $\mathrm{D}_{\mathrm{G}}(V)$ is smooth.

Denote by $\pi: V \rightarrow V / G$ the quotient map. There is a functor:

$$
\pi_{*}^{\mathrm{G}}: \mathrm{D}_{\mathrm{G}}(V) \rightarrow \mathrm{D}(V / \mathrm{G})
$$

where $\mathscr{F} \rightarrow \mathscr{F} \mathrm{G}$ is the functor of G-invariants. The left adjoint to $\pi_{*}^{\mathrm{G}}$ is $\mathbf{L} \pi^{*}$, where the $\mathrm{G}$ action on $\mathbf{L} \pi^{*} \mathscr{F}$ for any $\mathscr{F}$ in $\mathrm{D}(V / \mathrm{G})$ is the trivial one.

By construction of the quotient $V / \mathrm{G}$, we have $\pi_{*}^{\mathrm{G}} \mathscr{O}_{V}=\mathscr{O}_{V / \mathrm{G}}$. The projection formula implies that:

$$
\pi_{*}^{\mathrm{G}} \mathbf{L} \pi^{*} \simeq i d .
$$

The functor $\mathbf{L} \pi^{*}$ always commutes with arbitrary direct sums, whereas $\pi_{*}^{\mathrm{G}}$ commutes with arbitrary direct sums because it is right adjoint to $\mathbf{L} \pi^{*}$ and $\mathrm{D}_{\mathrm{G}}(V)$ is compactly generated. The inclusion $\pi_{*}^{\mathrm{G}} \mathrm{D}^{\mathrm{b}}{ }_{\mathrm{G}}(V) \subset \mathrm{D}^{\mathrm{b}}(V / \mathrm{G})$ comes from the properness of $\pi$.

Let me now study the right adjoint to $\mathbf{L} \pi^{*}$. The dualizing line bundle of $V$ is Gequivariantly locally trivial, hence $\omega_{V}=\pi^{*} \omega_{V / G}$. Thus, by Grothendieck duality, we have:

$$
\operatorname{Hom}_{\mathrm{D}^{\mathrm{b}}(V / \mathrm{G})}\left(\pi_{*} \mathscr{G}, \mathscr{F}\right)=\operatorname{Hom}_{\mathrm{D}^{\mathrm{b}}(V)}\left(\mathscr{G}, \mathbf{L} \pi^{*} \mathscr{F}\right),
$$

for all $\mathscr{G} \in \mathrm{D}^{\mathrm{b}}(V)$ and all $\mathscr{F} \in \mathrm{D}^{\text {perf }}(V / \mathrm{G})$. As a consequence, for any $\mathscr{G} \in \mathrm{D}^{\mathrm{b}}(V)$, we have:

$$
\begin{aligned}
\pi_{*} \mathbf{R} \mathscr{H} \operatorname{om}\left(\mathscr{G}, \mathscr{O}_{V}\right) & =\pi_{*} \mathbf{R} \mathscr{H} \operatorname{om}\left(\mathscr{G}, \mathbf{L} \pi^{*} \mathscr{O}_{V / \mathrm{G}}\right) \\
& =\mathbf{R} \mathscr{H} \operatorname{om}\left(\pi_{*} \mathscr{G}, \mathscr{O}_{V / \mathrm{G}}\right) .
\end{aligned}
$$

If $\mathscr{G}$ is assumed to be G-equivariant, then:

$$
\mathbf{R} \mathscr{H} \operatorname{om}\left(\pi_{*} \mathscr{G}, \mathscr{O}_{V / \mathrm{G}}\right)^{\mathrm{G}}=\mathbf{R} \mathscr{H} \operatorname{om}\left(\pi_{*}^{\mathrm{G}} \mathscr{G}, \mathscr{O}_{V / \mathrm{G}}\right) .
$$

From the above equalities, we find:

$$
\begin{aligned}
& \operatorname{Hom}_{\mathrm{D}^{\mathrm{b}}(V / \mathrm{G})}\left(\pi_{*}^{\mathrm{G}} \mathscr{G}, \mathscr{F}\right)=\operatorname{Hom}_{\mathrm{D}^{\mathrm{b}}(V / \mathrm{G})}\left(\mathscr{O}_{V / \mathrm{G}}, \mathbf{R} \mathscr{H} o m\left(\pi_{*}^{\mathrm{G}} \mathscr{G}, \mathscr{O}_{V / \mathrm{G}}\right) \otimes \mathscr{F}\right) \\
& =\operatorname{Hom}_{\mathrm{D}^{\mathrm{b}}(V / \mathrm{G})}\left(\mathscr{O}_{V / \mathrm{G}}, \pi_{*}^{\mathrm{G}} \mathbf{R} \mathscr{H} \operatorname{om}\left(\mathscr{G}, \mathscr{O}_{V}\right) \otimes \mathscr{F}\right) \\
& =\operatorname{Hom}_{\mathrm{D}^{\mathrm{b}}(V / \mathrm{G})}\left(\mathscr{O}_{V / \mathrm{G}}, \pi_{*}^{\mathrm{G}}\left(\mathbf{R} \mathscr{H} \operatorname{om}\left(\mathscr{G}, \mathscr{O}_{V}\right) \otimes \mathbf{L} \pi^{*} \mathscr{F}\right)\right) \\
& =\operatorname{Hom}_{\mathrm{D}^{\mathrm{b}}\left(\operatorname{Coh}^{\mathrm{G}}(V)\right.}\left(\mathscr{O}_{V}, \mathbf{R} \mathscr{H} \operatorname{om}\left(\mathscr{G}, \mathscr{O}_{V}\right) \otimes \mathbf{L} \pi^{*} \mathscr{F}\right) \\
& =\operatorname{Hom}_{\mathrm{D}^{\mathrm{b}}\left(\operatorname{Coh}^{\mathrm{G}}(V)\right)}\left(\mathscr{G}, \mathbf{L} \pi^{*} \mathscr{F}\right)
\end{aligned}
$$


for all $\mathscr{G} \in \mathrm{D}^{\mathrm{b}}{ }_{\mathrm{G}}(V)$ and all $\mathscr{F} \in \mathrm{D}^{\text {perf }}(V / \mathrm{G})$. Hence, $\mathbf{L} \pi^{*}$ is a right adjoint to $\pi_{*}^{\mathrm{G}}$, welldefined on $\mathrm{D}^{\text {perf }}(V / \mathrm{G})$. This already proves that $\pi_{*}^{\mathrm{G}}: \mathrm{D}_{\mathrm{G}}(V) \rightarrow \mathrm{D}(V / \mathrm{G})$ is a categorical weakly crepant resolution of singularities of $V / \mathrm{G}$. I go on demonstrating that it is in fact a strongly crepant resolution of singularities.

The category $\mathrm{D}_{\mathrm{G}}(V)$ has obviously a $V / \mathrm{G}$ module structure as it is naturally endowed with a tensor product. So I am only left to prove that the identity is a relative Serre functor for $\mathrm{D}^{\mathrm{b}}{ }_{\mathrm{G}}(V)$ with respect to $\mathrm{D}^{\mathrm{b}}(V / \mathrm{G})$. Let $\mathscr{G}_{1}$ and $\mathscr{G}_{2}$ be in $\mathrm{D}^{\mathrm{b}}{ }_{\mathrm{G}}(V)$, we have:

$$
\begin{aligned}
\mathbf{R} \pi_{*}^{\mathrm{G}} \mathbf{R} \mathscr{H} \operatorname{om}\left(\mathscr{G}_{1}, \mathscr{G}_{2}\right) & =\mathbf{R} \pi_{*}^{\mathrm{G}} \mathbf{R} \mathscr{H} \operatorname{om}\left(\mathscr{G}_{1} \otimes \mathbf{R} \mathscr{H} \operatorname{om}\left(\mathscr{G}_{2}, \mathscr{O}_{V}\right), \mathscr{O}_{V}\right) \\
& =\mathbf{R} \pi_{*}^{\mathrm{G}} \mathbf{R} \mathscr{H} \operatorname{om}\left(\mathscr{G}_{1} \otimes \mathbf{R} \mathscr{H} \operatorname{om}\left(\mathscr{G}_{2}, \mathscr{O}_{V}\right), \pi^{!} \mathscr{O}_{V / \mathrm{G}}\right) \\
& =\mathbf{R} \mathscr{H} \operatorname{om}\left(\mathbf{R} \pi_{*}^{\mathrm{G}}\left(\mathscr{G}_{1} \otimes \mathbf{R} \mathscr{H} \operatorname{om}\left(\mathscr{G}_{2}, \mathscr{O}_{V}\right)\right), \mathscr{O}_{V / \mathrm{G}}\right) \\
& =\mathbf{R} \mathscr{H} \operatorname{om}\left(\mathbf{R} \pi_{*}^{\mathrm{G}}\left(\mathbf{R} \mathscr{H} \operatorname{om}\left(\mathscr{G}_{2}, \mathscr{G}_{1}\right)\right), \mathscr{O}_{V / \mathrm{G}}\right) .
\end{aligned}
$$

This shows that the identity is indeed a relative Serre functor for $\mathrm{D}^{\mathrm{b}}{ }_{\mathrm{G}}(V)$ with respect to $\mathrm{D}^{\mathrm{b}}(V / \mathrm{G})$.

I want to prove that to prove that $\mathrm{D}_{\mathrm{G}}(V)$ is non-commutative in the sense of Van den Bergh, i.e. there exists a sheaf of algebras $\mathscr{A}$ on $V / G$ such that:

$$
\mathrm{D}^{\mathrm{b}}{ }_{\mathrm{G}}(V) \simeq \mathrm{D}^{\mathrm{b}}(V / \mathrm{G}, \mathscr{A}),
$$

and that $\mathscr{A}$ is a sheaf of Cohen-Macaulay $\mathscr{O}_{V / \mathrm{G}}$-modules. Let $V_{\rho_{1}}, \ldots, V_{\rho_{m}}$ be all the finite irreducible representations of $\mathrm{G}$. Let's prove that:

$$
\mathscr{G}:=\mathscr{O}_{V} \otimes V_{\rho_{1}} \oplus \cdots \oplus \mathscr{O}_{V} \otimes V_{\rho_{m}}
$$

is a tilting bundle for $\mathrm{D}^{\mathrm{b}}{ }_{\mathrm{G}}(V)$ with respect to $\pi_{*}^{\mathrm{G}}$. Notice that the functor $\pi_{*}^{\mathrm{G}}$ is exact, so that I only have to check that for any equivariant coherent sheaf $\mathscr{F}$, the vanishing $\pi_{*}^{\mathrm{G}} \mathscr{H} \operatorname{om}(\mathscr{G}, \mathscr{F})=0$ implies $\mathscr{F}=0$.

Let $\mathscr{F}$ be G-equivariant sheaf such that $\pi_{*}^{\mathrm{G}} \mathscr{H} \operatorname{om}(\mathscr{G}, \mathscr{F})=0$. The morphism $\pi$ is flat, the functor $\pi_{*}$ is exact and $\mathscr{G}$ is locally free, hence by base change, this is equivalent to:

$$
\mathscr{H} \operatorname{om}\left(\mathscr{G}, \mathscr{F} \otimes \mathscr{O}_{\pi^{-1}(y)}\right)^{\mathrm{G}}=0,
$$

for all $y \in V / \mathrm{G}$. We have a G-equivariant surjection $\mathscr{G} \rightarrow \mathscr{G} \otimes \mathscr{O}_{\pi^{-1}(y)}$, so that the above vanishing implies:

$$
\mathscr{H} \operatorname{om}\left(\mathscr{G} \otimes \mathscr{O}_{\pi^{-1}(y)}, \mathscr{F} \otimes \mathscr{O}_{\pi^{-1}(y)}\right)^{\mathrm{G}}=0 .
$$

But $\mathscr{O}_{\pi^{-1}(y)}$ is a finite $\mathbb{C}$-algebra and $\mathscr{F}$ is a G-equivariant coherent sheaf. Hence, by Schur's lemma, we find $\mathscr{F} \otimes \mathscr{O}_{\pi^{-1}(y)}=0$ for all $y \in V / \mathrm{G}$, that is $\mathscr{F}=0$. I thus proved that $\mathscr{G}$ is a tilting bundle for $\mathrm{D}^{\mathrm{b}}{ }_{\mathrm{G}}(V)$ with respect to $\pi_{*}^{\mathrm{G}}$. We deduce that (see [9]):

$$
\mathrm{D}_{\mathrm{G}}^{\mathrm{b}}(V) \simeq \mathrm{D}^{\mathrm{b}}\left(V / \mathrm{G}, \pi_{*}^{\mathrm{G}} \mathscr{E} n d(\mathscr{G})\right) .
$$

The relative Serre functor of $\pi_{*}^{\mathrm{G}}: \mathrm{D}_{\mathrm{G}}^{\mathrm{b}}(V) \rightarrow \mathrm{D}^{\mathrm{b}}(V / \mathrm{G})$ is the identity. Hence, the proof of lemma 4.8 of [10] shows that the sheaf $\pi_{*}^{\mathrm{G}} \mathscr{E} n d(\mathscr{G})$ is a sheaf of Cohen-Macaulay $\mathscr{O}_{V / \mathrm{G}^{-}}$ modules.

Corollary 3.0.13 Let $V$ be a finite dimensional vector space and let $\mathrm{G}$ be a finite subgroup of $\mathrm{SL}(V)$. Then $V / \mathrm{G}$ admits a non-commutative strongly crepant resolution in the sense of Van den Bergh.

This corollary already appeared many times in the literature (see [20]). Note that our construction precisely gives the expected algebra for the non-commutative resolution of $V / \mathrm{G}$, that is the skew-algebra $\operatorname{Sym} V \# G$. 
Proof Indeed, if $\mathrm{G}$ is a subgroup of SL( $V)$, then $\mathrm{G}$ acts trivially on the volume form of $V$. This implies that $\omega_{V}$ is G-equivariantly trivial, so that we can apply the above result.

I now deal with my second main result:

Theorem 3.0.14 Let $X$ be a quasi-projective variety with normal Gorenstein quotient singularities and let $\mathscr{X}$ be a smooth separated Deligne-Mumford stack whose coarse moduli space is X. Assume that the dualizing line bundle of $\mathscr{X}$ is the pull back of the dualizing line bundle on $X$, then $\mathrm{D}(\mathscr{X})$ is a strongly crepant resolution of $X$.

Furthermore, there exists a sheaf of algebras $\mathscr{A}$ on $X$ such that $\mathrm{D}^{\mathrm{b}}(\mathscr{X}) \simeq \mathrm{D}^{\mathrm{b}}(X, \mathscr{A})$. Hence, the pair $(X, \mathscr{A})$ is a non-commutative crepant resolution of $X$ in the sense of Van den Bergh.

Note that if $X$ is a normal quasi-projective variety with quotient singularities, then there is always a smooth separated Deligne-Mumford stack associated to it as in the above statement (see proposition 2.8 of [21]). The non-trivial hypothesis (which can not be removed) is that the dualizing bundle of the Deligne-Mumford stack associated to $X$ is the pull back of the dualizing bundle on $X$. This amounts to check that on an étale atlas of $\mathscr{X}$, the line bundle $\omega_{\mathscr{X}}$ is equivariantly ${ }^{1}$ locally trivial. This finally comes down to verify that for any $x \in X$, there exists an étale neighborhood $U_{x}$ of $x \in X$, such that $U_{x}=V / \mathrm{G}$ where $V$ is a vector space and $\mathrm{G}$ acts on $V$ as a subgroup of $\operatorname{SL}(V)$.

Proof This proof follows exactly the same pattern as the proof of Theorem 1.0.2. However, in the present case, I have to use some delicate results about Deligne-Mumford stacks in order to have the same machinery work.

The category $\mathrm{D}(\mathscr{X})$ is certainly cocomplete. By [18], corollary 5.2 , the category $\mathrm{D}(\mathscr{X})$ is also compactly generated. Since $\mathscr{X}$ is a smooth and separated Deligne-Mumford stack and $\mathrm{D}(\mathscr{X})$ is compactly generated, a small variation on lemma 3.27 in [19] shows that $\mathrm{D}(\mathscr{X})$ is smooth.

Let $\pi: \mathscr{X} \rightarrow X$ be the projection from $\mathscr{X}$ to its coarse moduli space. By construction of the coarse moduli space of a Deligne-Mumford stack, we know that $\pi_{*}$ is exact and that $\pi_{*} \mathscr{O}_{\mathscr{X}}=\mathscr{O}_{X}$. Hence, by the projection formula, we have:

$$
\pi_{*} \mathbf{L} \pi^{*} \simeq i d,
$$

where $\mathbf{L} \pi^{*}$ is the left adjoint to $\pi_{*}$. The functor $\mathbf{L} \pi^{*}$ always commutes with arbitrary direct sums, whereas $\pi_{*}$ commutes with arbitrary direct sums because it is right-adjoint to $\mathbf{L} \pi^{*}$ and $\mathrm{D}^{\mathrm{b}}(\mathscr{X})$ is compactly generated. Finally, the morphism $\pi$ is proper, so that $\pi_{*} \mathrm{D}^{\mathrm{b}}(\mathscr{X}) \subset$ $\mathrm{D}^{\mathrm{b}}(X)$.

Let me show that the relative Serre functor of $\mathrm{D}^{\mathrm{b}}(\mathscr{X})$ with respect to $\mathrm{D}^{\mathrm{b}}(X)$ is trivial. By Grothendieck duality for Deligne-Mumford stack (see [16], Theorem 2.27), the right adjoint to $\pi_{*}$ is equal to $\mathbf{L} \pi^{*} \otimes \omega \mathscr{X} \otimes \pi^{*} \omega_{X}^{-1}$ when restricted to $\mathrm{D}^{\text {perf }}(X)$. This already proves that $\mathrm{D}(\mathscr{X})$ is a categorical weakly crepant resolution of $X$. But the category $\mathrm{D}(\mathscr{X})$ is endowed with a tensor product, so that the same basic computations as in the proof of Theorem 1.0.2 show that the relative Serre functor $\mathrm{D}^{\mathrm{b}}(\mathscr{X})$ with respect to $\mathrm{D}^{\mathrm{b}}(X)$ is indeed trivial.

I am left to prove that the exists a tilting vector bundle for $\mathrm{D}^{\mathrm{b}}(\mathscr{X})$ with respect to $\pi$. But $\pi_{*}$ is exact, so I only have to prove the existence of a vector bundle $\mathscr{G}$ on $\mathscr{X}$ such that for any coherent sheaf $\mathscr{F}$ on $\mathscr{X}$, the vanishing $\pi_{*} \mathscr{H}$ om $(\mathscr{G}, \mathscr{F})=0$ implies $\mathscr{F}=0$. The existence of such a vector bundle is exactly the content Theorem 4.4 and Theorem 5.3 of [13].

${ }^{1}$ For the isotropy groups of the fixed points of the étale atlas of $\mathscr{X}$. 
Lemma 4.8 of [10] allows again to conclude that $\pi_{*} \mathscr{E} n d(\mathscr{G})$ is a sheaf of Cohen-Macaulay $\mathscr{O}_{X}$-modules.

\section{Some connections with the McKay correspondence}

In this section, I make some connections between the theory of categorical resolutions and the McKay correspondance. I have proved that for any smooth quasi-projective variety $V$ acted on by a finite group $\mathrm{G}$ such that $\omega_{V}$ is G-equivariantly locally trivial, the derived category $\mathrm{D}_{\mathrm{G}}(V)$ is a strongly crepant resolution of $V / \mathrm{G}$. In this situation, Conjecture 4.10 of [14] can be restated as follows:

Conjecture 4.0.15 Let $V$ be a smooth quasi-projective variety and let $\mathrm{G}$ be a finite subgroup of $\operatorname{Aut}(V)$ such that $\omega_{V}$ is G-equivariantly locally trivial. Then, for any resolution of singularities $Z \rightarrow V / \mathrm{G}$, there exists an admissible fully faithful embedding:

$$
\mathrm{D}_{\mathrm{G}}^{\mathrm{b}}(V) \hookrightarrow \mathrm{D}^{\mathrm{b}}(Z) .
$$

In particular, if $Z$ is a crepant resolution of $V / G$, then there is an equivalence:

$$
\mathrm{D}_{\mathrm{G}}^{\mathrm{b}}(V) \simeq \mathrm{D}^{\mathrm{b}}(Z) \text {. }
$$

The last part of this conjecture has been proved in [4] if $\operatorname{dim} V \leq 3$ or if $V$ is symplectic and $\mathrm{G}$ acts by symplectic automorphisms. This result is known as the categorical McKay correspondence. I refer to [7] for some discussions on the global version of the last part of this conjecture. We will prove a very special case of it in the context of cyclic groups acting by dilations on finite dimensional vector spaces.

Theorem 4.0.16 Let $V$ be a vector space of dimension $n$ and let $\mathrm{G}$ be a cyclic group of order $d$ acting by dilations on $V$ (with d dividing $n$ ). Then we have an equivalence:

$$
\mathrm{D}_{\mathrm{G}}^{\mathrm{b}}(V) \simeq \mathscr{T}_{0}
$$

where $\mathscr{T}_{0}$ is the categorical strongly crepant resolution of $V / \mathrm{G}$ constructed by Kuznetsov (see Theorem 2.0.11). In particular, there is a fully faithful embedding:

$$
\mathrm{D}_{\mathrm{G}}^{\mathrm{b}}(V) \hookrightarrow \mathrm{D}^{\mathrm{b}}(\widetilde{V / \mathrm{G}})
$$

where $\widetilde{V / G}$ is the resolution of $V / G$ obtained by blowing-up 0 . In the special case where $d=n$ (so that $\widetilde{V / G}$ is a crepant resolution of $V / G$ ), there is an equivalence:

$$
\mathrm{D}_{\mathrm{G}}^{\mathrm{b}}(V) \simeq \mathrm{D}^{\mathrm{b}}(\widetilde{V / \mathrm{G}}),
$$

If $\mathrm{G}$ is a cyclic group acting on $V$ by dilations, the blow-up of $V / \mathrm{G}$ along 0 is the "smallest" geometric resolution of singularities known for $V / G$. Hence, in that specific case, I believe that Theorem 4.0.16 should render a proof of Conjecture 4.0.15 tractable.

Proof We denote $X:=V / \mathrm{G}$ and $q: \tilde{X} \rightarrow X$ the blow-up of $X$ along 0 . Let $q: \tilde{V} \rightarrow V$ the blow-up of along 0 and consider the projection $p_{*}^{G}: \mathrm{D}^{\mathrm{b}}\left(\operatorname{Coh}^{\mathrm{G}}(\tilde{V}) \rightarrow \mathrm{D}^{\mathrm{b}}(\tilde{X})\right.$, where $p: \tilde{V} \rightarrow \tilde{X}$ is the quotient map and $\mathscr{F} \rightarrow \mathscr{F} \mathrm{G}$ is the functor of invariants. 
As $V$ is smooth, the category $\mathrm{D}^{\mathrm{b}}{ }_{\mathrm{G}}(V)$ is a full admissible subcategory of $\mathrm{D}^{\mathrm{b}}{ }_{\mathrm{G}}(\tilde{V})$. Let $\chi_{1}, \ldots, \chi_{d-1}$ be the characters of $\mathrm{G}$. The same argument as in the proof of Theorem 1.0.2 shows that the vector bundle:

$$
\mathscr{O}_{\tilde{V}} \otimes \chi_{0} \oplus \cdots \oplus \mathscr{O}_{\tilde{V}} \otimes \chi_{d-1}
$$

is a tilting bundle for $\mathrm{D}_{\mathrm{G}}^{\mathrm{b}}(V)$ with respect to $p_{*}^{\mathrm{G}}$. Recall that $\tilde{X}$ is the total space of $t: \mathscr{O}_{\mathbb{P}^{n-1}}(-d) \rightarrow \mathbb{P}^{n-1}$. The quotient map $p: \tilde{V} \rightarrow \tilde{X}$ is a $d$ to 1 cover ramified along the zero section of $t$. Hence, we have:

$$
p_{*} \mathscr{O}_{\tilde{V}}=t^{*} \mathscr{O}_{\mathbb{P}^{n-1}}(-d+1) \oplus \cdots \oplus t^{*} \mathscr{O}_{\mathbb{P}} n
$$

In particular

$$
p_{*}^{\mathrm{G}}\left(\mathscr{O}_{\tilde{V}} \otimes \chi_{j}\right)=t^{*} \mathscr{O}_{\mathbb{P}^{n-1}}(-j) .
$$

Since we have the vanishing $\mathbf{R}^{i} q_{*} t^{*} \mathscr{O}_{\mathbb{P} n-1}(-j)$ for all $1-d \leq j \leq d-1$ and all $i>0$, we deduce that $\mathscr{O}_{\tilde{V}} \otimes \chi_{0} \oplus \cdots \oplus \mathscr{O}_{\tilde{V}} \otimes \chi_{d-1}$ is a tilting bundle for $\mathrm{D}_{\mathrm{G}}^{\mathrm{b}}(V)$ with respect to $q_{*} p_{*}^{\mathrm{G}}$. As a consequence, we have:

$$
\mathrm{D}^{\mathrm{b}}{ }_{\mathrm{G}}(V) \simeq \mathrm{D}^{\mathrm{b}}\left(X, q_{*} p_{*}^{\mathrm{G}}\left(\mathscr{E} n d\left(\mathscr{O}_{\tilde{V}} \otimes \chi_{0} \oplus \cdots \oplus \mathscr{O}_{\tilde{V}} \otimes \chi_{d-1}\right)\right)\right) .
$$

From the computations just above, we immediately find that:

$$
\begin{aligned}
& q_{*} p_{*}^{\mathrm{G}}\left(\mathscr{E} n d\left(\mathscr{O}_{\tilde{V}} \otimes \chi_{0} \oplus \cdots \oplus \mathscr{O}_{\tilde{V}} \otimes \chi_{d-1}\right)\right) \\
& \quad=q_{*} \mathscr{E} n d\left(t^{*} \mathscr{O}_{\mathbb{P}^{n-1}}(-d+1) \oplus \cdots \oplus t^{*} \mathscr{O}_{\mathbb{P}^{n-1}}\right) .
\end{aligned}
$$

We then deduce that:

$$
\mathrm{D}_{\mathrm{G}}^{\mathrm{b}}(V) \simeq \mathscr{T}_{0}
$$

where $\mathscr{T}_{0}$ is the categorical strongly crepant resolution of $X$ constructed by Kuznetsov (see Theorem 2.0.11).

If $d=n$, one notices that $\mathscr{T}_{0}=\mathrm{D}^{\mathrm{b}}(\tilde{X})$, so that $\mathrm{D}_{\mathrm{G}}^{\mathrm{b}}(V) \simeq \mathrm{D}^{\mathrm{b}}(\tilde{X})$.

Acknowledgments I am very grateful to Richard Thomas for mentioning and suggesting to me to prove the aforementioned results on categorical strongly crepant resolutions of quotient singularities. I would also like to thank Tom Bridgeland for sharing his unpublished preprint [6] with me, Andrea Petracci for some useful advice on Deligne-Mumford stacks and Enrica Floris for helpful comments on a preliminary version of this paper.

Open Access This article is distributed under the terms of the Creative Commons Attribution 4.0 International License (http://creativecommons.org/licenses/by/4.0/), which permits unrestricted use, distribution, and reproduction in any medium, provided you give appropriate credit to the original author(s) and the source, provide a link to the Creative Commons license, and indicate if changes were made.

\section{References}

1. Abuaf, R.: Dualité projective homologique et résolutions catégoriques des singularités. Thèse pour le grade de Docteur es Sciences de l'Université de Grenoble (2013)

2. Abuaf, R.: Wonderful resolutions and categorical resolutions of singularities. J. Reine Angew. Math. (2013). doi:10.1515/crelle-2013-0104

3. Abuaf, R.: Categorical crepant resolutions of singularities and the Tits-Freudenthal magic square (2013). arXiv: 1307.1675

4. Bridgeland, T., King, A., Reid, M.: The McKay correspondence as an equivalence of derived categories. J. Am. Math. Soc. 14(3), 535-554 (2001) (electronic) 
5. Bondal, A., Orlov, D.: Derived categories of coherent sheaves. In: Li, T.T. et al. (eds.) Proceedings of the International Congress of Mathematicians, ICM 2002, Beijing, China, August 20-28, 2002. Vol. II: Invited Lectures, pp. 47-56. Beijing: Higher Education Press (2002)

6. Bridgeland, T.: Derived Categories and Minimal Models. Unpublished manuscript (2004)

7. Chen, J.-C., Tseng, H.-H.: A note on derived McKay correspondence. Math. Res. Lett. 15(3), 435-445 (2008)

8. Fujiki, A.: On resolutions of cyclic quotient singularities. Publ. Res. Inst. Math. Sci. 10(1), 293-328 $(1974 / 75)$

9. Hille, L., Van den Bergh, M.: Fourier-Mukai transforms. In: Handbook of Tilting Theory, volume 332 of London Mathematics Society Lecture Note Series, pp. 147-177. Cambridge University Press, Cambridge (2007)

10. Iyama, O., Wemyss, M.: On the noncommutative Bondal-Orlov conjecture. J. Reine Angew. Math. 683, 119-128 (2013)

11. Kawamata, Y.: Derived categories and birational geometry. In: Algebraic Geometry-Seattle 2005. Part 2, volume 80 of Proceedings of Symposium Pure Mathematics, pp. 655-665. American Mathematical Society, Providence (2009)

12. Kuznetsov, A., Lunts, V.: Categorical resolutions of irrational singularities (2012). arXiv:1212.6170

13. Kresch, A.: On the geometry of Deligne-Mumford stacks. In: Algebraic Geometry-Seattle 2005. Part 1, volume 80 of Proceedings of Symposium Pure Mathematics, pp. 259-271. American Mathematical Society, Providence (2009)

14. Kuznetsov, A.: Lefschetz decompositions and categorical resolutions of singularities. Selecta Math. 13(4), 661-696 (2008)

15. Neeman, A.: The Grothendieck duality theorem via Bousfield's techniques and Brown representability. J. Am. Math. Soc. 9(1), 205-236 (1996)

16. Nironi, F.: Grothendieck duality for Deligne-Mumford stacks (2008). arXiv:0811.1955

17. Peskin, B.R.: On the dualizing sheaf of a quotient scheme. Commun. Algebra 12(15-16), 1855-1869 (1984)

18. Toën, B.: Derived Azumaya algebras and generators for twisted derived categories. Invent. Math. 189(3), $581-652$ (2012)

19. Toën, B., Vaquié, M.: Moduli of objects in dg-categories. Ann. Sci. École Norm. Sup. 40(3), 387-444 (2007)

20. Van den Bergh, M.: Three-dimensional flops and noncommutative rings. Duke Math. J. 122(3), 423-455 (2004)

21. Vistoli, A.: Intersection theory on algebraic stacks and on their moduli spaces. Invent. Math. 97(3), 613-670 (1989) 